\title{
VASCULAR RISK FACTORS AND COGNITIVE FUNCTIONS IN THE PATIENTS WITH CEREBROVASCULAR DISEASE
}

10.36740/WLek202010128

\author{
Oksana 0. Kopchak ${ }^{1,2}$, Natalia Yu. Bachinskaya ${ }^{1,2}$, Oleksandr R. Pulyk ${ }^{3}$ \\ 'KYIV MEDICAL UNIVERSITY, KYIV, UKRAINE \\ ${ }^{2}$ CHEBOTAREV STATE INSTITUTE OF GERONTOLOGY OF THE NATIONAL ACADEMY OF MEDICAL SCIENCES OF UKRAINE, KYIV, UKRAINE \\ ${ }^{3}$ DEPARTMENT OF NEUROREHABILITATION, UZHHOROD NATIONAL UNIVERSITY, UZHHOROD, UKRAINE
}

\begin{abstract}
The aim: To assess the severity of cognitive impairment (Cl) in patients with cerebrovascular disease (CVD) depending on the number of vascular risk factors (VRF). Materials and methods: The study consisted of five hundred and eighty patients with CVD (247 females and 333 males) aged from 45 to 89 years (mean age: 64,1 $\pm 8,9$ years). The patients were divided into 6 groups $(I, I I, I I I, I V, V, V I)$ depending on the number of VRF. The examination consisted of a standard clinical evaluation, neurological examination, the application of neuropsychological tests (the MMSE; the PALT/The Paired Associates Learning Test; clock drawing test), laboratory tests, MRI of brain.

Results: According to ANOVA, the greater the number ofVRF the patients had, the lower was their total MMSE score corresponding to more pronounced cognitive decline ( $F=2,97$, $p=0,012$ ). A significant negative correlation between patients' age and their MMSE score was detected regardless of their VRF count. The patients of the V and VI groups had substantially lower parameters of immediate, delayed memory and clock drawing test score comparing to the patients of the I group.

Conclusions: The presence of 4 and more VRF was related to more pronounced Cl in the patients with CVD. Decline of different aspects of memory, attention, spatial orientation, abstract thinking, planning, concentration, executive and visuospatial skills in groups of patients with 4,5 and 6 vascular risk factors was established. Age was substantially associated with cognitive decline in all the group of patients.
\end{abstract}

KEY WORDS: vascular risk factors, cognitive impairment, cerebrovascular disease

Wiad Lek. 2020;73(10):2250-2254

\section{INTRODUCTION}

Aging of population all over the world is strongly associated with a high prevalence of chronic, non-communicable diseases including cognitive decline and dementia [1,2]. There is growing recognition of the role of vascular risk factors (VRF) in the development of cognitive impairment (CI) [3]. Majority of cardiovascular risk factors have recently been associated with cognitive decline; cardiovascular health is now identified as a crucial claim for optimal brain functioning [4]. Nowadays modifiable risk factors for CI are well determined $[5,6]$. These, in particular, contain smoking, low physical activity, sedentary lifestyle, poor diet, excessive alcohol consumption, midlife obesity, high blood pressure, midlife high cholesterol and diabetes [7]. It is found that such cardiovascular risk factors as diabetes mellitus, hypertension, hypercholesterolemia, atrial fibrillation, smoking are linked to the development of both vascular dementia and Alzheimer disease [8]. Some studies have estimated the role of distinct risk factors in patients' cognitive decline. Hense, adiposity in late life has even demonstrated an obvious protective effect against $\mathrm{CI}$; a meta-analysis suggested that underweight as well as overweight/obesity in midlife increased dementia risk $[9,10]$. In the study of Ganguli et al., elevated total cholesterol was substantially connected with better performance in memory tests. They also found complex relationships with blood pressure (BP): elevated diastolic pressure was associated with worse baseline performance in executive and language functions, but at the same time was protective against deterioration in language. No significant association was found between systolic BP/history of hypertension and baseline cognitive performance, but it was defensive against decline in memory. As with cholesterol, a similar J-shaped relationship has been illustrated for BP increase in midlife versus late life in relation to risk of late-life dementia [3]. The association between multiple vascular risk factors and the cognitive decline has not been clearly established.

\section{THE AIM}

Our study aimed to assess the severity of cognitive impairment (CI) in patients with cerebrovascular disease (CVD) depending on the number of vascular risk factors (VRF).

\section{MATERIALS AND METHODS}

The current study consisted of five hundred and eighty patients with CVD ( 247 females and 333 males) aged from 45 to 89 years (mean age: $64,1 \pm 8,9$ years). 


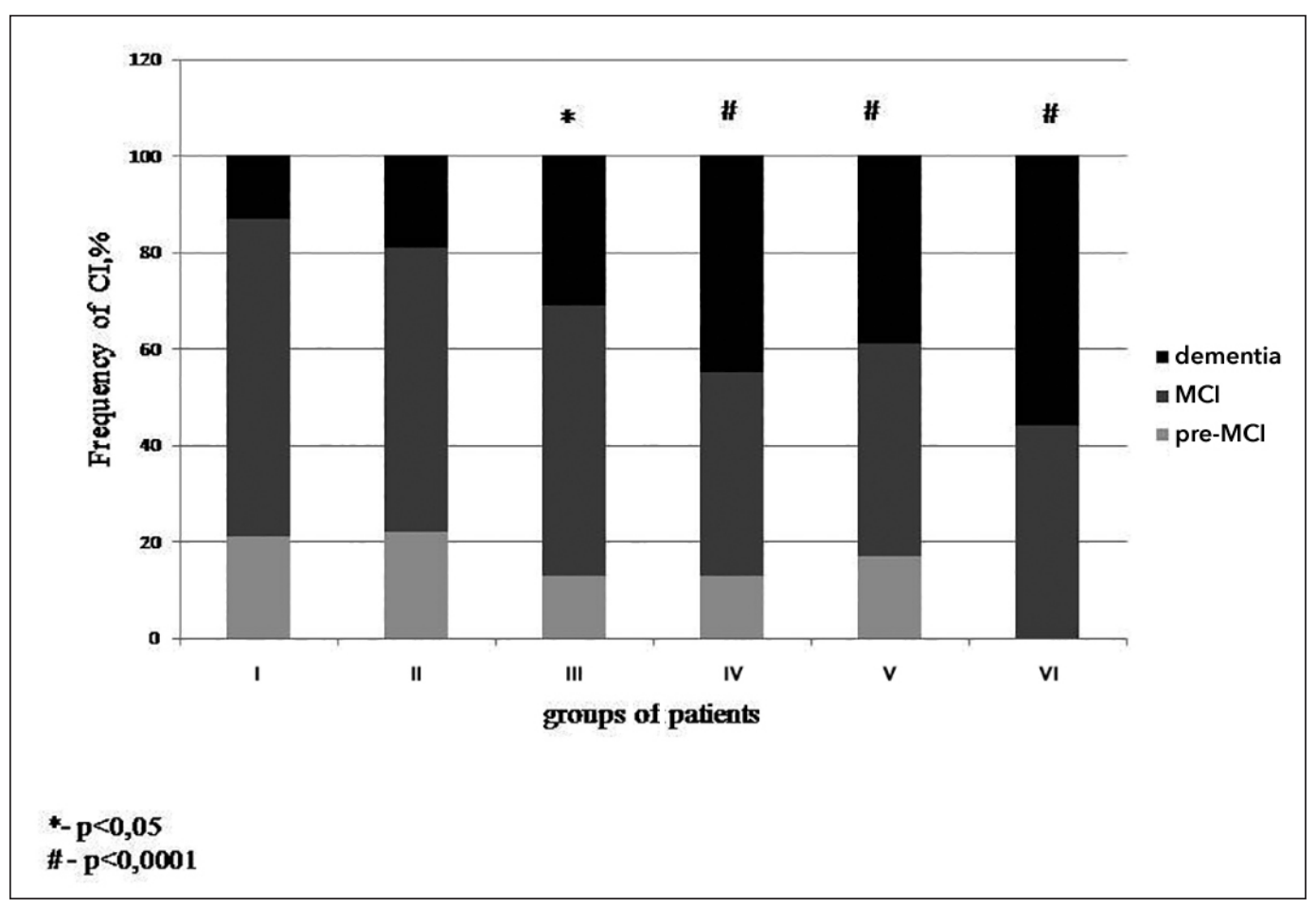

Fig.1. Frequency of pre-MCl, $\mathrm{MCl}$ and dementia in all the groups of patients
The patients were divided into 6 groups depending on the number of VRF:

I group consisted of 71 patients with arterial hypertension/AH);

II- 147 patients with two VRF: obesity and AH;

III - 158 patients with three VRF: obesity, AH, diabetes mellitus/DM;

IV - 133 patients with four VRF: obesity, AH, DM, increased serum triglyceride level/ISTL;

V- 46 patients with five VRF, including obesity, AH, DM, ISTL, lower level of high density lipoproteins/LLHDL;

VI- 25 patients with six VRF, including obesity, AH, DM, ISTL, LLHDL and high level of lower density lipoproteins (HLLDL).

A medical history was obtained from all the participants. The examination consisted of a standard clinical evaluation, including anthropometric examination (body mass index - BMI, waist circumference), neurological examination, the application of neuropsychological tests, laboratory tests (complete blood count, biochemical parameters, blood lipid profile, serum thyroid hormones), MRI of brain. To evaluate patients' cognitive functions the following tests were used: the MMSE/Mini- Mental State Examination (assessing patient's ability in time and place orientation, short-term, long-term memory status, speech function, gnosis, praxis) [11]; the PALT/The Paired Associates Learning Test (depicting state of the immediate and delayed recognition memory on the verbal stimuli [12]; clock drawing test (evaluates short term memory, understanding of verbal instructions, spatial orientation, abstract thinking, planning, concentration, executive and visuospatial skills) [13]. According to MMSE scores: 29-30 - absence of CI, 28-27 - pre-mild CI (pre-MCI), 25-26 - mild CI (MCI), $\leq 24$ points - dementia (D).
Recruitment criteria were as follows: patients 45 years and older with signs of cerebrovascular disease and presence of the following risk factors: obesity, $\mathrm{AH}, \mathrm{DM}$, ISTL, LLHDL and high level of lower density lipoproteins (HLLDL).

Participants were excluded if they were younger than 45 , had history of stroke, tumors, disease of thyroid gland; were too ill to participate, had severe vision or hearing impairments, were incapacitated.

All the subjects provided written informed consent. Ethical approval (Protocol No.1 from 11 of January 2020) was obtained from the Bioethics Commission of the Chebotarev State Institute of Gerontology of the National Academy of Medical Sciences of Ukraine.

Student's t-test $(\mathrm{t})$ was applied for evaluating credibility between mean quantitative positions of two samples. Proportions were compared using $\chi 2$. The Pearson's correlation coefficient ( $r$ ) between different indicators was analyzed. A value of $p<0,05$ was considered statistically significant.

\section{RESULTS AND DISCUSSION}

The main complaints of the patients were the following: decline of memory and attention to recent events, current events, names, dates, and so on, vocabulary difficulties, inability to concentrate, decreased occupational performance, increased irritability, general weakness and increased fatigue, decreased physical and mental performance. Neurological examination revealed pyramidal signs, pathological reflexes, impaired coordination (intention tremor, ataxia, missing the mark), sensory disorders (in particular in the distal parts of limbs in the form of creeps, numbness, especially in patients with diabetes mellitus). 
Table 1. Severity of $\mathrm{Cl}$ in clinical groups

\begin{tabular}{cccc}
\hline Groups & MMSE score & $P$ (in comparison to the patients of the I group) & Significance \\
\hline$I(n=71)$ & $24,68 \pm 0,28$ & & $N S$ \\
\hline$I I(n=147)$ & $24,78 \pm 0,16$ & $P=0,77$ & $\mathrm{NS}$ \\
\hline$I I I(n=158)$ & $24,23 \pm 0,19$ & $P=0,18$ & $\mathrm{~S}$ \\
\hline$I V(n=133)$ & $23,59 \pm 0,25$ & $P=0,007$ & $\mathrm{~S}$ \\
\hline$V(n=46)$ & $23,15 \pm 0,43$ & $P=0,003$ & $\mathrm{~S}$ \\
\hline$V I(n=25)$ & $21,48 \pm 0,54$ & $P<0,0001$ & \\
\hline
\end{tabular}

NS- non significant, S- significant

According to MMSE the frequency of pre-MCI, MCI and dementia (D) in the group was as follows:

I (pre-MCI - 15/21\%; MCI - 47/66\%; D - 9/13\%);

II (pre-MCI - 32/22\%; MCI - 87/59\%; D - 28/19\%);

III (pre-MCI - 21/13\%; MCI - 88/56\%; D - 49/31\%);

IV (pre-MCI - 17/13\%, MCI - 56/42\%; D - 60/45\%);

V (pre-MCI - 8/17\%; MCI - 20/44\%; D -18/39\%);

VI (pre-MCI - 0/0\%; MCI - 11/44\%; D -14/56\%).

The frequency of dementia was significantly higher in patients of the III (31\%), IV (45\%), V (39\%) and VI (56\%) groups comparing to the I group participants $(13 \%)$ (correspondently: $\chi^{2}=10,1, p<0,05 ; \chi^{2}=24,9, p<0,0001 ; \chi^{2}=17,8$, $\mathrm{p}<0,0001 ; \chi^{2}=52,2, \mathrm{p}<0,0001$ ) (Fig.1). Among the patients of the VI group no individuals with Pre-MCI were found.

According to obtained results pre-mild CI was only noticeable for the patients themselves and sometimes for family members. Pre-MCI was determined by memory deficits (both subjective and from an extensive clinical interview) and normal performance on several neuropsychological tests, including the MMSE [14]. They observed a decline in cognitive activity compared to their usual previous experience and normal MMSE score. In particular, patients began to spend more time searching for the necessary information in memory, they somewhat slowed down the mental operations necessary to sort out everyday professional tasks. In addition, some patients mentioned difficulty in concentration. It became more difficult to keep a steady focus on performing a particular operation or to switch from one object to another. Patients also told about minor memory impairments, which, however, did not affect their ability to navigate time and space.

MCI was manifested as impairment of memory, attention or ability to study, and were confirmed by the results of neuropsychological test. Nevertheless, the group of patients with MCI was characterized by maintaining complete autonomy in their daily lives. Patients complained of increased fatigue while performing mental work. Most patients with MCI demonstrated deterioration in multiple cognitive domains: thinking, attention, mental performance, information processing speed, memory in comparison with the average age norm. However, their MMSE parameters did not reach the level of dementia.

Clinical diagnosis of dementia was established in accordance with ICD 11, taking into consideration the criteria requiring memory disorders (impaired ability to remember new material, to reproduce previously learned information) associated with the decline in at least one additional cognitive domain (impaired judgment, thinking, planning and processing information), emotional and motivational disorders that lead to changes in social functioning and daily activity. In the presence of dementia, patients completely or partially lost their independence and often needed care. In the presence of mild dementia, patients had limited professional and social activity, but mostly retained the ability to live independently, maintain personal hygiene. In moderate dementia, patients experienced difficulties in living independently; they constantly needed control and care; the MMSE total score was $\leq 19$ points. Among the examined patients there were no persons with severe dementia with impaired activity in daily life, inability to maintain minimal personal hygiene and impaired motor abilities, and with necessity for constant care.

We assessed the severity of CI in all clinical groups (IIVI) in comparison to the results of the I group according to the MMSE (Tab.1).

Patients from the IV, V and VI groups with number of VRF from 4 to 6 had significantly lower MMSE score comparing to those with one vascular risk factor. According to ANOVA, the greater the number of VRF the patients had, the lower was their total MMSE score corresponding to more pronounced cognitive decline $(\mathrm{F}=2,97, \mathrm{p}=0,012)$.

In all the groups we found significant negative correlation between the age of patients and total MMSE score (I group: $r=-0,63, p<0,001 ;$ II: $r=-0,35, p<0,001$; III group: $\mathrm{r}=-0,37, \mathrm{p}<0,001$; IV group: $\mathrm{r}=-0,38, \mathrm{p}<0,001 ; \mathrm{V}$ group: $\mathrm{r}=-0,49, \mathrm{p}<0,001$; VI group: $\mathrm{r}=-0,54, \mathrm{p}<0,001)$. Thus, a substantial negative correlation between patients' age and their MMSE score was established regardless of their VRF count.

The patients of the V and VI groups had substantially lower parameters of PALT immediate memory comparing to the patients of the I group (correspondently: $18,67 \pm 1,05$ vs $21,23 \pm 0,71, p=0.039 ; 17,12 \pm 1,25$ vs $21,23 \pm 0,71, p=0.004$ ). Concerning PALT delayed memory, participants of the IV, V and VI groups showed considerably lower score in comparison to those of the I group (correspondently: $32,48 \pm 1,54$ (IV group) vs $38,82 \pm 1,93$ (I group), $\mathrm{p}=0.014$; $30,93 \pm 2,52$ (V group) vs $38,82 \pm 1,93$ (I group), $\mathrm{p}=0.013$; $29,36 \pm 3,24$ (VI group) vs $38,82 \pm 1,93$ (I group), $\mathrm{p}=0.02$ ). In other groups no significant difference concerning PALT parameters was found comparing to the patients of the I group $(\mathrm{p}>0,05)$. 
The clock drawing test results revealed substantial difference in the participants of the V and VI groups comparing to the patients of the first group (correspondently: $7,73 \pm 0,38$ (V group) vs $8,66 \pm 0,18$ (I group), $\mathrm{p}=0.02$; $6,40 \pm 0,37$ (VI group) vs $8,66 \pm 0,18$ (I group), $\mathrm{p}<0.0001$ ).)

Consequently, the higher was the number of risk factors (4 and more) in our patients, the more significant was their cognitive decline. This finding is in accordance with other studies.

In particular, in the large community-based cohort change in cognitive performance was associated with treatable vascular risk in both middle-aged and old people [15]. This vascular risk was based on the components of the Framingham Risk Score for Cardiovascular Disease (FRS-CD) that are responsive to treatment: diabetes mellitus (yes/no), current smoker status (yes/no), systolic blood pressure ( $\mathrm{mmHg}$ ), total cholesterol ( $\mathrm{mmol} / \mathrm{l})$, HDL cholesterol $(\mathrm{mmol} / \mathrm{l})$ and use of blood pressure lowering drugs (yes/no). The FRS-CD was conceived to foresee the risk of a new cardiovascular, cerebrovascular or peripheral vascular episode within the next ten years. A higher treatable vascular risk score was connected with a greater risk of a new vascular event: the lowest score was -5 (10-year risk $<1 \%$ ), and the highest one was 21 (10-year risk >30\%) [16]. van Eersel et al. suggested that the influence of treatable vascular risk on cognitive performance was comparable to the impact of age. They support the hypothesis that the onset of vascular risk management at late-life may be too late for successful prevention of cognitive decline and dementia [15]. According to our results, a significant negative correlation between patients' age and their MMSE score was detected regardless of their VRF count. However, our study differs from this one in study population, number of participants; it was not a longitudinal one. At the same time, we included greater amount of vascular risk factors. Our results were comparable to the data of the two studies from the National Alzheimer Coordinating Center (NACC) cohort, in which low cognitive performance was linked with high treatable vascular risk independent of age $[17,18]$. In a systematic review by Peters et al., an apparent relationship between the exposure to greater numbers of baseline risk factors and an elevated risk of later cognitive impairment or incident dementia was found [7]. These data somehow match our findings that the presence of 4 and more VRF is associated with more pronounced cognitive decline in the patients with CVD. At the same time, this study has some advantages and strengths, including Cochrane based Methodology, detailed search terms and successful inclusion of the data resulting in description of study populations from 18 studies and 9 countries across Europe, Australia and North America with $>40000$ participants and follow-up from midlife and late life [7].

In the Atherosclerosis Risk in Communities (ARIC) Study, which is an ongoing project, midlife diabetes, hypertension, obesity and hypercholesterolemia were connected with later life mild cognitive impairment and dement [5]. Ganguli et al. in the population sample study found that several vascular risk factors (history of stroke, diabetes, central obesity, C-Reactive Protein), although associated with lower baseline cognitive accomplishment, did not predict the level of subsequent decline. At the same time, $A P O E^{\star} 4$ genotype was connected with advanced deterioration in language, memory, and executive functions. Homocysteine elevation was associated with more pronounced decline in executive function. Hypertension (history or systolic blood pressure $>140 \mathrm{~mm}$ ) was linked with slower drop in memory. Baseline alcohol consumption was associated with slower decline in attention, language, and memory [3]. These findings don't concur with our results, where we underline deterioration of cognitive functions of our patients in several cognitive domains in case of combination of more than 4 VRF. The data of Ganguli et al., differ from our study in the large number of participants, age of patients and cardiovascular risk factors. In particular we did not assess the influence of $A P O E^{\star} 4$ genotype, homocysteine elevation. The authors also used a comprehensive test battery tapping the cognitive domains of attention/processing speed, executive function, memory, language, and visuospatial functions, but our tests don't coincide.

Finally, our results are in line with data of other studies $[7 ; 15]$ that support the necessity for both clinicians and patients to reduce the risk factor exposure and in those cases where risk factors are well indicated to prevent their further augmentation.

\section{CONCLUSIONS}

The presence of 4 and more VRF was related to more pronounced $\mathrm{CI}$ in the patients with CVD. In particular, we established decline of different aspects of memory, impairment of attention, spatial orientation, abstract thinking, planning, concentration, executive and visuospatial skills in groups patients with 4,5 and 6 vascular risk factors. Age was substantially associated with cognitive decline in all the group of patients. The results of our study emphasize on the importance of powerful control of vascular risk factor exposure to prevent possible cognitive deterioration in patients with cerebrovascular disease.

\section{REFERENCES}

1. Lourenco J., Serrano A., Santos-Silva A. et al. Cardiovascular Risk Factors Are Correlated with Low Cognitive Function among Older Adults Across Europe Based on The SHARE Database. Aging and disease. 2018;9(1):90-101.

2. Morovic S., Budincevic H., GovoriV., Demarin V. Possibilities of Dementia Prevention - It is Never Too Early to Start. Journal of Medicine and Life. 2019; 2(4): 332-337. D0I: 10.25122/jml-2019-0088

3. Ganguli M., Fu B., Snitz B.E. et al. Vascular risk factors and cognitive decline in a population sample. Alzheimer Dis Assoc Disord. 2014;28(1): 9-15. doi:10.1097/WAD.0000000000000004

4. Gorelick P.B., Furie K.L., ladecola C. et al. Defining optimal brain health in adults: a presidential advisory from the American Heart Association/ American Stroke Association. Stroke. 2017; 48: e284-e303.

5. Knopman D.S., Gottesman R.F., Sharrett A. R. Midlife vascular risk factors and midlife cognitive status in relation to Prevalence of Mild Cognitive Impairment and Dementia in later life: the ARIC Study. Alzheimers Dement. 2018 ; 14(11): 1406-1415. doi:10.1016/j.jalz.2018.03.011. 
6. Lincoln P., Fenton K., Alessi C. et al. The Black friars Consensus on brain health and dementia. The Lancet. 2014;383:1805-6.

7. Peters R., Booth A., Rockwood K. et al. Combining modifiable risk factors and risk of dementia: a systematic review and meta-analysis. BMJ Open. 2019;9:e022846. doi:10.1136/ bmjopen-2018-022846.

8. Solomon A., Turunen H., Ngandu T. et al. Effect of the Apolipoprotein E Genotype on Cognitive Change During a Multidomain Lifestyle Intervention: A Subgroup Analysis of a Randomized Clinical Trial. JAMA Neurol. 2018;75(4):462-470.

9. Luchsinger J.A., Biggs M.L., Kizer J.R. et al. Adiposity and Cognitive Decline in the Cardiovascular Health Study. Neuro epidemiology. 2013; 40:274-281. [PubMed: 23445925]

10. Anstey K.J., Cherbuin N., Budge M., Young J. Body mass index in midlife and late-life as a risk factor for dementia: a meta-analysis of prospective studies. Obes Rev. 2011; 12:e426-37. [PubMed: 21348917]

11. Folstein M., Folstein S., McHugh P.R. Mini-mental state: a practical method for grading the cognitive state of patients for the clinical. Journal of Psychiatric Research 1975; 12: 189-198.

12. Parmenter B.A., Denney D.R., Lynch S.G. The cognitive performance of patients with multiple sclerosis during periods of high and low fatigue. Multiple Sclerosis 2003; 9(2): 111-118. https://doi.org/ 10.1191/1352458503ms8590a.

13. Freedman M., Leach L., KaplanE. etal.Clock-drawing: a neuropsychological analysis. New York, NY: Oxford University Press, 1994.

14. van Eersel1 M.E.A., Joosten H., Gansevoort R.T., Slaets J.P.J., Izaks G.J. Treatable Vascular Risk and Cognitive Performance in Persons Aged 35 Years or Older: Longitudinal Study of Six Years. J Prev Alz Dis.2019;1(6):42-49 http://dx.doi.org/10.14283/jpad.2018.47.

15. Jefferson A.L., Hohman T.J., Liu D. et al. Adverse vascular risk is related to cognitive decline in older adults. J Alzheimers Dis. 2015;44(4):1361-1373.

16. Viswanathan A., Macklin E.A., Betensky R. et al. The influence of vascular risk factors and stroke on cognition in late life: Analysis of the NACC cohort. Alzheimer Dis Assoc Disord. 2015;29(4):287-293.
17. Peters R., Booth A., Rockwood K. et al. Combining modifiable risk factors and risk of dementia: a systematic review and meta-analysis. BMJ Open 2019;9:e022846. doi:10.1136/ bmjopen-2018-022846

18. Knopman D.S., Gottesman R.F., Sharrett A.R. et al. Midlife vascular risk factors and midlife cognitive status in relation to Prevalence of Mild Cognitive Impairment and Dementia in later life: the ARIC Study. Alzheimers Dement. 2018 November ; 14(11): 1406-1415. doi:10.1016/j.jalz.2018.03.011

\section{ORCID and contributionship:}

Oksana O. Kopchak: 0000-0003-2666-0616,

Natalia Yu. Bachinskaya: 0000-0001-6199-1416

Oleksandr R. Pulyk: 0000-0002-8717-047X E,F

\section{Conflict of interest:}

The Authors declare no conflict of interest

\section{CORRESPONDING AUTHOR Oksana 0. Kopchak}

Neurology, Psychiatry and Physical Rehabilitation

Kyiv Medical University, Ukraine

e-mail:dr.kopchak@kmu.edu.ua

Received: 23.04 .2020

Accepted: 03.09 .2020

A - Work concept and design, B - Data collection and analysis, C - Responsibility for statistical analysis, D-Writing the article, $\mathbf{E}$-Critical review, $\mathbf{F}$ - Final approval of the article 